\title{
Bibliographie du dossier « Des langues vivantes à l'école »
}

\section{Marie-Hélène Lemoine}

\section{OpenEdition}

1 Journals

Édition électronique

URL : http://journals.openedition.org/ries/3408

DOI : $10.4000 /$ ries.3408

ISSN : 2261-4265

Éditeur

Centre international d'études pédagogiques

Édition imprimée

Date de publication : 1 mars 1996

Pagination : 135-139

ISSN : 1254-4590

\section{Référence électronique}

Marie-Hélène Lemoine, «Bibliographie du dossier « Des langues vivantes à l'école » », Revue

internationale d'éducation de Sèvres [En ligne], 09 | 1996, mis en ligne le 20 août 2013, consulté le 23

mars 2021. URL : http://journals.openedition.org/ries/3408 ; DOI : https://doi.org/10.4000/ries.3408

Ce document a été généré automatiquement le 23 mars 2021.

(c) Tous droits réservés 


\title{
Bibliographie du dossier « Des langues vivantes à l'école »
}

\author{
Marie-Hélène Lemoine
}

1 Cette bibliographie propose une sélection des publications de ces cinq dernières années. Les références traitées collectivement, en particulier les numéros spéciaux de revues ont été placées en première partie, mais leur contenu est aussi constitué d'études de cas relevant de la deuxième partie.

\section{Questions de pédagogie et de didactique : vers une formation des enseignants}

2 Des ouvrages de fond ont vu le jour (O'Neill, Luc) et les principales revues de didactique des langues ont toutes consacré un numéro spécial à ce thème. Il faut aussi mentionner les travaux des cinq ateliers du Conseil de l'Europe sur ce sujet (disponibles sur simple demande au service Langues vivantes du Conseil de l'Europe).

3 ARNSDORF Dieter (coord.), BOYLE Helen (coord.), CHAIX Paul (coord.), O'NEIL Charmian (coord.), «L'apprentissage des langues étrangères à l'école primaire $=$ Fremdsprachenlernen in der Grundschule = Foreign Language Learning in the Primary School $»$. Triangle, $n^{\circ} 11,1992,136 p$. Des experts européens posent la question des enjeux de l'apprentissage d'une langue étrangère à l'école primaire : quels enseignants ou intervenants? Apprentissage ou sensibilisation? Quelle liaison avec le secondaire? Quels objets introduire dans l'apprentissage? Les activités créatives s'opposent-elles à une progression grammaticale des acquisitions? En même temps, ce numéro fait le point sur certains contextes (français, allemand) et propose des activités pédagogiques en général (raconter des histoires) et une progression pour l'anglais.

4 Conseil de l'Europe, Learning and Teaching Languages in Pre-School and Primary Bilingual Contexts (Age 3/4 - 12/13). Report on Workshop 5A, Carmarthen, Wales (United Kingdom), 16-21 September 1991, $248 \mathrm{p}$.

Cet atelier s'intéresse aux différents types d'éducation bilingue et dans ce cadre, à 
l'interface enseignement pré-élémentaire/enseignement élémentaire, à la formation des enseignants, aux moyens d'enseignement. Les études de cas concernent le pays de Galles et la Catalogne. Atelier 5B (juin 95), à paraître.

5 Conseil de l'Europe, Foreign Language Education in Primary Schools (Age 5-11). Report on Workshop 8A, Locum, Germany, 17-22 May 1992, 136 p.

Une variété de comptes rendus de travaux de groupes (curriculum, continuité, méthodologie, supports, formation permanente des enseignants), une synthèse des approches et de nombreuses communications portant sur les pratiques des pays suivants : Allemagne, Finlande, Écosse, Pays-Bas, États-Unis. Par ailleurs le programme $8 \mathrm{~A}$ s'intéresse à la formation des enseignants à l'université et à l'utilisation des livres pour enfants. Atelier 8B (mai 1995), à paraître.

Conseil de l'Europe, Apprentissage et enseignement des langues vivantes à l'école primaire. Rapport de l'atelier 4B, Sèvres, France, 12-17 décembre 1993, $254 \mathrm{p}$.

Les travaux de cet atelier du Conseil de l'Europe ont sont ici rapportés en trois parties : méthodologie, évaluation, formation des enseignants, avec les documents de travail correspondants.

7 Conseil de l'Europe, The Challenge of In-Service Trainlng for Foreign Language Teachers in Primary Schools. Atelier 17, septembre 1995, Spain (à paraitre).

DE VRIENDT M.-J., "Initiation précoce à une langue étrangère: un jeu d'enfant... Peut-être, mais... ». Revue de phonétique appliquée, $n^{\circ}$ 107, 1993, p. 113-124, bibliogr.

Cet article repose sur des échanges et des réflexions faites lors de l'accompagnement d'expériences d'initiation d'élèves de 3 à 8 ans au néerlandais ou à l'anglais qui prouvent que malgré l'hétérogénéité des parcours et du fonctionnement, il y a homogénéité dans le discours relatif aux pratiques de classe et une convergence dans les demandes des enseignants. Trois pratiques sont expliquées: la centration sur l'apprenant, l'approche ludique, les interactions en langue orale. Enfin la question est posée de la formation des enseignants.

8 GARABÉDIAN Michèle (dir.), "Enseignements/apprentissages précoces des langues». Le français dans le monde (Recherches et applications), septembre 1991, p. 6-192.

Dans ce numéro très complet se succèdent des analyses des politiques linguistiques en la matière (France, Suisse, Europe) ainsi qu'une présentation de la question des curricula, une discussion des principaux concepts en cause (éveil aux langages, perception auditive, transdisciplinarité) avec des considérations de psychopédagogie et de neuropédagogie, une analyse de pédagogies à l'œuvre (Louisiane, Italie, Madagascar, Brésil, Tessin, Yougoslavie, Écosse, Catalogne et Allemagne), et enfin un inventaire analytique de matériels didactiques.

GARABÉDIAN Michèle (dir.), "Quels modèles didactiques pour enseigner/apprendre une langue étrangère à de jeunes scolaires? ", Études de linguistique appliquée, n 89, mars 1993, 124 p., bibliogr.

Les principaux spécialistes du moment sont rassemblés ici, chacun apportant son éclairage (et une bibliographie) pour ce numéro. Sont proposés tout autant des exposés didactiques théoriques (Michèle Garabédian, Christiane Luc, Élisabeth Calaque, Danièle Moore) qu'un tour d'horizon en contexte (l'anglais à Liège, contexte bilingue du Val d'Aoste, formation linguistique des instituteurs écossais par les modules vidéo d'« Une vie d'instit ») et différents moyens d'enseignement (le jeu-langage, l'image, le poème). Institut national de recherche pédagogique, département Didactiques des disciplines, Paris, 
INRP, 1991, 226 p., bibliogr.

Ce premier grand colloque français aborde les aspects épistémologiques, didactiques et institutionnels de l'enseignement des langues à l'école élémentaire avec de nombreux spécialistes.

11 LUC Christiane, Approche d'une langue étrangère à l'école. 1 Perspectives sur l'apprentissage. 2 : Étude psycholinguistique et aspects didactiques. Paris, INRP, 1992, 100 p. +180 p., bibliogr.

Premier rapport consacré à la recherche menée par l'INRP sur « une préparation à l'enseignement des langues vivantes à l'école élémentaire ». Le deuxième volume contient une réflexion didactique et une étude de cas psycholinguistique sur l'appropriation par les élèves de CM2 d'un système linguistique en anglais et en allemand, en ce qui concerne le genre grammatical.

MALLET Bernard (coord.), "Enseigner le français langue étrangère à l'école primaire et maternelle : recherches et propositions ». Lidil, n 4, mai 1991, 156 p., bibliogr.

Ce numéro regroupe à la fois des réflexions théoriques concluant à la nécessité d'un effort méthodologique et de formation considérable pour réussir cet enseignement, des études de cas (Italie, Val d'Aoste), et des comptes rendus de praticiens, notamment en ce qui concerne l'utilisation de l'image et l'enseignement de la civilisation.

13 O'NEIL Charmian, Les enfants et l'enseignement des langues étrangères. Saint-Cloud, France, CREDIF (Centre de recherche et d'étude pour la diffusion du français), Paris, HatierDidier, 1993, 287 p., bibliogr.

Cet ouvrage offre un panorama très étendu de l'histoire des expérimentations dans de nombreux pays monolingues et dans toutes les situations rencontrées (langue minoritaire, enfants de migrants, enseignement international), avec une place privilégiée pour la France. Il présente en outre une synthèse des caractéristiques du public du point de vue affectif, cognitif, ainsi que des stratégies utilisées par les adultes, dans leurs interactions avec les élèves. L'auteur analyse enfin les tendances et interrogations suscitées dans ce domaine.

\section{Questions de politique linguistique : études de cas en Europe}

COLE Amy G., "Foreign Language Teaching in the Primary School in Scotland". Intercompreensao, $n^{\circ} 3$, septembre 1993, p. 59-65.

Description des projets pilotes dans les écoles primaires écossaises.

EMERT Karl (dir.), Frühes Fremdsprachenlernen: Schulreform für Europa. RehburgLoccum, Allemagne, Evangelische Akademie Loccum, 1992, $284 \mathrm{p}$.

Un tableau dressé en 1990 et du point de vue allemand de l'enseignement précoce des langues en Europe : arrière-plan théorique, enjeux, méthodes.

Enseigner les langues vivantes à l'école élémentaire, Colloque national 25-26 janvier 1993, Clermont-Ferrand. Clermont-Ferrand, France, CRDP, 1993, $263 p$.

De multiples interventions pour faire le point sur l'enseignement précoce des langues vivantes dans les académies françaises et dans d'autres régions d'Europe (les Länder allemands par exemple) ainsi que sur les échanges possibles. L'occasion a été donnée également à des institutions (sous-direction de la Coopération linguistique et éducative, CNDP) de présenter leur rôle dans la mise en place de l'EPLV. 
17 L'enseignement précoce des langues en Europe à l'horizon 2000 : bilans et perspectives. Saint-Gervais, France, CMIEB (Centre mondial d'information sur l'éducation bilingue), Besançon, France, CLAB (Centre de linguistique appliquée), 1992, 243 p.

Ce colloque était organisé en trois thèmes : études de cas (méthodologies et résultats des actions d'enseignement/apprentissage précoce et d'éducation bilingue); formation des enseignants; apprentissages précoces et enjeux de société. À l'intérieur de ces thèmes, chaque atelier a fait l'objet d'un rapport par les spécialistes du domaine.

18 "La place des langues étrangères dans les systèmes éducatifs de l'Union européenne", in: Les chiffres clés de l'éducation européenne 94. Luxembourg, Office des publications officielles des Communautés européennes, 1995, p. 72-80.

Ce chapitre présente un bref aperçu historique de la situation, puis, sous forme de nombreux tableaux comparatifs, il indique les pourcentages d'élèves apprenant les langues aux niveaux primaire et secondaire, ainsi que le temps qui y est consacré. Enfin il résume rapidement les dispositifs de formation initiale des enseignants dans les deux niveaux.

19 "L'apprentissage des langues vivantes étrangères en Europe", in: Rapport de l'Inspection générale de l'Éducation nationale 1995. Paris, Documentation française, 1995, p. 353-431.

Ce rapport est un ensemble de monographies sur les onze pays qui, avec la France, constituaient en 1994 l'Union européenne. Chacune d'entre elles aborde la question des langues à l'école primaire (ou enseignement fondamental) puisque celles-ci y ont actuellement leur place dans chacun des systèmes éducatifs des pays concernés. Le cas de la France n'est pas traité ici.

20 PERREGAUX Christiane, "L'école, espace plurilingue ", in: "Jalons pour une Europe des langues ». Lidil, avril 1995, p. 125-139.

L'auteur développe ici une recherche menée dans des classes maternelles suisses, essentiellement pluriculturelles, sur l'éveil aux langages: elle donne ses références théoriques et des exemples d'activités pédagogiques. Il s'agit de briser le monolinguisme de la classe et de favoriser une attitude d'ouverture aux langues.

21 PICHI PIAZZA Elena, "La lingua straniera nella scuola elementare : problemi organizzativi e didattici ». Scuola e citta, $n^{\circ} 11$, novembre 1992, p. 496-500.

Intégration de l'enseignement de la langue étrangère à l'école élémentaire, ses modalités, le langage non verbal, dans le cadre d'une «alphabétisation culturelle » et de l'« unité de l'enseignement ».

Programme deuxième langue (enseignement primaire) : français, néerlandais, allemand. Bruxelles, Belgique, ministère de l'Éducation nationale et de la Culture française, [1992], 48 p.

La Belgique est un des premiers pays à avoir rédigé des programmes et instructions pour l'enseignement d'une langue étrangère à l'école (appelée ici deuxième langue). Les principes, finalités, techniques de classe sont ici communes aux trois langues.

TSCHOUMY Jacques-André, Parler européen demain? Institut roman de recherches et de documentation pédagogiques, s.l., Suisse, Romandie de l'éducation Euro-Région, Suisse, [1994], 50 p., bibliogr.

Dans cet ouvrage composé de cinq dossiers, le troisième intitulé «Vers un enseignement/apprentissage plus précoce encore", porte sur l'idée que le plurilinguisme suisse peut servir d'exemple pour la réflexion sur le thème du plurilinguisme européen. La mise en œuvre d'un enseignement précoce des langues est 
décrite de façon très concrète, les nombreuses difficultés également, mais le projet est irréversible.

\section{Questions de politique linguistique : le cas de la France}

BOBASCH Michaëla, «L'enseignement des langues à l'école primaire: enquête». Le Monde de l'éducation, n²21, décembre 1994, p. 28-42.

Diverses initiatives concernant l'apprentissage des langues à l'école primaire se sont développées au cours des dernières années. Depuis 1989, avait été lancée une expérimentation contrôlée d'enseignement des langues vivantes au cours moyen. Le Nouveau contrat pour l'école envisage, à partir de la rentrée 1995, une initiation généralisée pour tous les élèves de cours élémentaire première année. Mais le problème du suivi et celui de la formation des enseignants restent posés.

Enquêtes dans les écoles publiques et privées du premier degré: langues vivantes étrangères à l'école, tableaux statistiques. Paris, ministère de l'Éducation nationale, DEP, juin 1995.

Académie par académie, des statistiques à propos du nombre d'élèves (par niveaux), des langues enseignées, des intervenants, des outils utilisés, des relations avec le pays de la langue étudiée. (Ce document est synthétisé dans la Note d'information, DEP, $n^{\circ}$ 95-46.) "L'enseignement des langues vivantes à l'école élémentaire", in: Rapport de l'Inspection générale de l'Éducation nationale 1993. Paris, Documentation française, 1993, p. 257-281.

Ce rapport clôt la période de trois ans d'enseignement d'initiation aux langues étrangères à l'école primaire. Laissant de côté le point de vue didactique, il s'attache surtout aux problèmes relevés comme les plus aigus : d'une part la gestion (intégration de cet enseignement dans l'école et parmi les autres disciplines, organisation, fonctionnement des équipes de pilotage, continuité avec le collège), d'autre part la formation des intervenants.

O'NEIL Charmian, "Solution de continuité entre les niveaux. Étude de cas: le passage du primaire au secondaire en France ». Études de linguistique appliquée, $n^{\circ}$ 98, juin 1995, $p$. 85-91, bibliogr.

L'enseignement des langues dans le primaire en France illustre bien les problèmes qui se posent en l'absence d'un curriculum unifié, problèmes d'autant plus prévisibles depuis l'introduction d'un enseignement des langues dès le CE1. Cet article présente les suggestions que font à ce propos d'une part les enseignants impliqués dans cet enseignement, d'autre part l'institution, et conclut à la nécessité d'un curriculum intégré du primaire au secondaire.

De façon générale les trois sources suivantes, de périodicité annuelle, sont à consulter régulièrement car elles incluent souvent un chapitre sur l'enseignement des langues vivantes à l'école :

Rapport de l'Inspection générale de l'Éducation nationale, DEP-Documentation française La rentrée scolaire : dossier de presse, direction de la Communication, MENESR.

"Repères et références statistiques sur les enseignements et la formation» (DEP) : reprise partielle des données de la publication thématique Notes et études documentaires de la même source et traitant également annuellement de ce sujet. 
INDEX

Mots-clés : langues, langues étrangères, enseignement des langues 